

\section{Mortalidad Hospitalaria \\ en Hospital San Felipe, Año 2010}

*María Guadalupe Romero-Andrade

RESUMEN. La investigación se centra en el análisis de las causas de muerte hospitalaria en el año 2010 en el Hospital San Felipe, hospital de referencia nacional del sistema de salud de Honduras con cita en la ciudad de Tegucigalpa con el objetivo de mejorar la planificación de la atención sanitaria. Los datos señalan al cáncer como la principal causa de muerte hospitalaria, de tal forma que al sumarizar todas las causas, representa el 63.5\%, es decir, más de la mitad de la causas analizadas. Por procedencia el departamento de Francisco Morazán, representan más de la mitad de la casuística revisada (60.19\%). La distribución de mortalidad hospitalaria por sexo, en el caso de las mujeres es de $55.33 \%$ y en el caso de los hombres corresponde a $44.67 \%$. El rango de edad en el que ocurren estas muertes es de 0.5 días a 91 años y la media de edad del grupo es de 61.9 años.

Palabras Claves: mortalidad hospitalaria; tasa bruta de mortalidad; causas de muerte.

ABSTRACT. This investigation focus in the analysis of the causes of hospital death in 2010 in the San Felipe Hospital, the national referral hospital health system with appointment in the city of Tegucigalpa, Honduras with the objective to improve the planning of health care. The data indicate cancer as the leading cause of death in hospital, so that to summarize all causes, representing $63.5 \%$, more than half of the cases tested. By origin the Department of Francisco Morazán, representing more than half of the cases reviewed (60.19\%). The distribution of hospital mortality by sex, in the case of females is $55.33 \%$ and in the case of men corresponds to $44.67 \%$. The age range in which these deaths occur is 0.5 days to 91 years. The mean age of the group was 61.9 years.

Keywords: hospital mortality, crude death rate, death rate, causes of death.

\section{Introducción}

En los últimos años, la importancia de los datos hospitalarios ha crecido. Esto es resultado del papel que las estadísticas han logrado alcanzar a través del desarrollo de la capacidad informática, el incremento en el número de codificadores, la descentralización en la generación de los datos hospitalarios y el aumento en el uso de la información hospitalaria en la evaluación y planeación de los servicios (Salud, 2002).

A partir del año 1976, Rutsein y su equipo de colaboradores definieron una serie de afecciones hospitalarias que estaban sujetas, desde el punto de vista científico, de poder evitar la enfermedad, incapacidad y/o muerte a través de los medios hospitalarios disponibles, ya sean preventivos o curativos.
La mortalidad hospitalaria representa a su vez, uno de los indicadores de impacto conformando el grupo de indicadores de calidad que son monitorizados en los hospitales para la toma de decisiones tanto en la gestión, como en la planificación.

El Hospital San Felipe, es un hospital nacional con 130 años de servicio al país, cuenta en su interior con varios centros nacionales de referencia entre los que se pueden mencionar: cáncer, oftalmológico, rehabilitación. No cuenta con sala de emergencia para la atención de pacientes adultos y si cuenta con una sala de maternidad que atiende las 24 horas del día brindándose atenciones a pacientes con criterios de bajo riesgo.

La revisión de las causas de mortalidad del Hospital San Felipe, permite visualizar el comportamiento del patrón epidemiológico del paciente hospitalizado en el

Recibido: septiembre 2012 / aceptado y versión final octubre 2012.

* Msc. en Salud Pública, Msc. en Administración de Servicios de Salud, Canditada al Doctorado en Ciencias con Orientación en Ciencias de la Administración. 
año 2010 con lo cual, se plantean las disyuntivas para mejorar la calidad de la atención, la acción preventiva a nivel de atención primaria.

Teniendo como objetivo el de mejorar la planificación de la atención sanitaria, se aporta la información que se obtienen al analizar las defunciones ocurridas en el Hospital San Felipe en el año 2010.

\section{Metodología}

Se realizó una revisión retrospectiva utilizando como fuente de datos los que se generan en la Unidad de Estadística del Hospital y los expedientes de ese período.

Las variables que se utilizaron fueron: procedencia, edad (al momento de la muerte), sexo, causa básica de muerte y mes de ocurrencia de la muerte.

Los datos fueron obtenidos por personal del Departamento de Epidemiologia, en este caso, de Vigilancia de Infecciones Intrahospitalarias, registrándose en un instrumento que se diseñó para tal efecto y los datos se procesaron en el paquete Epi-Info 3.5.1.

\section{Resultados de Investigación}

Entre el 1 de enero y 31 de diciembre del año 2010, se registraron 103 defunciones. Todos los pacientes murieron en el hospital, ninguno llegó muerto.

En ese año se reportaron 15,918 egresos por lo que la tasa bruta de mortalidad hospitalaria fue de $0.647 \%$.

La velocidad de muerte por mes, es de 2 por semana y el mes que reportó más muertes fue el mes de abril con un promedio de 3 por semana.

El departamento de procedencia de los pacientes fallecidos se muestra en el Cuadro No.1 y se puede evidenciar que los pacientes que proceden del Departamento de Francisco Morazán, representan más de la mitad de la casuística revisada (60.19\%); el departamento de Valle, ocupa el segundo lugar (6.79\%), siguiendo en ese orden los departamentos de Comayagua y Choluteca que tienen similar dato (5.82\%); El Paraíso continúa en cuanto a porcentaje $(3.88 \%)$, presentando los departamentos de
Cortés y Olancho similar dato (2.91\%); lo mismo que Yoro y Atlántida (1.94\%); finalizan con el mismo porcentaje, los departamentos de Intibucá, Lempira, la Paz, Sta. Bárbara, Islas de la Bahía, Colón y Copán (0.94) (Cuadro No.1).

Cuadro No.1. Distribución se Acuerdo a Procedencia de Pacientes Fallecidos. Hospital San Felipe, 2010.

\begin{tabular}{|l|c|}
\hline Departamento & Promedio \\
\hline Valle & 6.79 \\
\hline Comayagua & 5.82 \\
\hline Choluteca & 5.82 \\
\hline El Paraiso & 3.88 \\
\hline Cortés & 2.91 \\
\hline Olancho & 2.91 \\
\hline Yoro & 1.94 \\
\hline Atlántida & 1.94 \\
\hline Intibucá & 0.94 \\
\hline Lempira & 0.94 \\
\hline La Paz & 0.94 \\
\hline Santa Bárbara & 0.94 \\
\hline Islas de La Bahia & 0.94 \\
\hline Colón & 0.94 \\
\hline Copán & 0.94 \\
\hline Total 16 & 0.94 \\
\hline
\end{tabular}

Fuente: Estadística de Hospital San Felipe

La distribución de mortalidad hospitalaria por sexo, se muestra en el Gráfico No.1 y en el caso de las mujeres es de $55.33 \%$ y en el caso de los hombres corresponde a $44.67 \%$.

El rango de edad en el que ocurren estas muertes es de 0.5 días a 91 años. La media de edad del grupo es de 61.9 años. La media y la mediana de edad por sexo, se

Gráfico No.1. Distribución por Sexo de Pacientes Fallecidos. Hospital San Felipe, Año 2010.

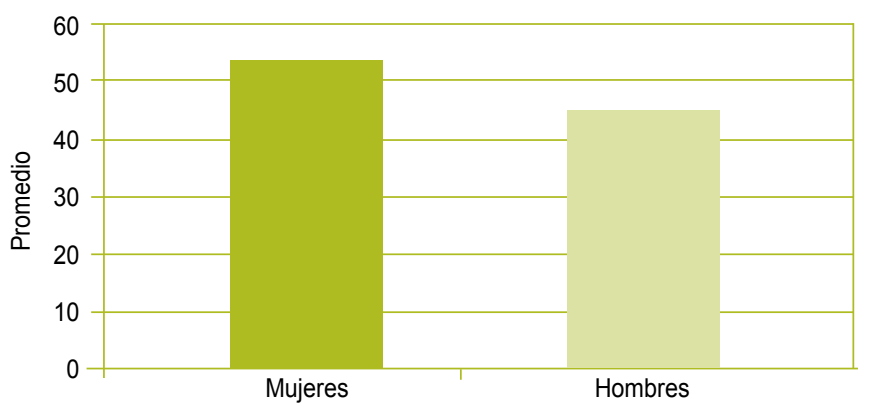

Fuente: Estadística de Hospital San Felipe. 
muestra en la Gráfica No.2, en donde se observa que las mujeres tienen una media de 57.67 años, una mediana de 60 años y en el caso de los hombres, tienen una media de 63.26 con una mediana de 67 años.

Gráfico No. 2. Distribución por Media y Mediana de la Edad de Pacientes Fallecidos. Hospital San Felipe. 2010.

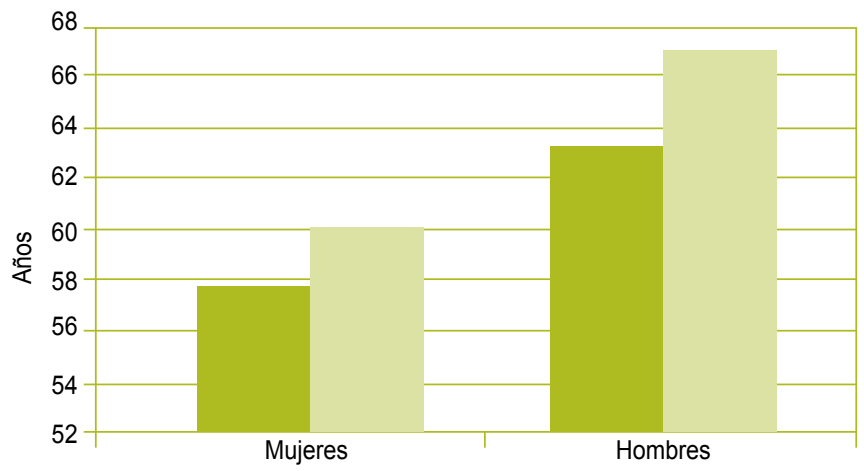

Fuente: Estadística de Hospital San Felipe.

Las principales causas de muerte ordenadas por frecuencia fueron: cáncer de mama y cáncer gástrico con 9.70\%; hepatocarcinoma con 8.73\%; insuficiencia respiratoria con $7.86 \%$; cáncer de recto y cirrosis hepática con $5.82 \%$; cáncer de colon y cáncer de esófago con 4.85\%; cáncer de pulmón con 3. 88\%; cáncer de útero, carcinoma metastásico, hipertensión arterial, desnutrición proteica del adulto y shoque séptico con $2.91 \%$; linfoma de Hodgkin, cáncer de páncreas, malformación congénita y alcoholismo crónico con 1.94\%; cáncer de parótida derecha, cáncer de vejiga, leucemia linfoblástica crónica, insuficiencia cardíaca congestiva, cáncer de vesícula, tumor de yeyuno, tumor retroperitoneal y enfermedad de Alzheimer con 0.97\% (Cuadro No.2).

\section{Discusión}

El análisis descriptivo de los datos de mortalidad que se reportaron en el Hospital San Felipe en el año 2010, debe tomarse en cuenta para los fines de la planificación de la atención tanto hospitalaria como a nivel de atención primaria.

La relación entre lo que es la atención curativa y la preventiva debe lograr un mayor sinergismo en aras de
Cuadro No.2. Distribución por Causa de Pacientes Fallecidos. Hospital San Felipe. Año 2010.

\begin{tabular}{|c|c|c|}
\hline Causa & No. & $\%$ \\
\hline Cáncer de Mama & 10 & 9.70 \\
\hline Cáncer gástrico & 10 & 9.70 \\
\hline Hepatocarcinoma & 9 & 8.73 \\
\hline Insuficiencia respiratoria & 8 & 7.76 \\
\hline Cáncer de recto & 6 & 5.82 \\
\hline Cirrosis hepática & 6 & 5.82 \\
\hline Cáncer de colon & 5 & 4.85 \\
\hline Cáncer de esófago & 5 & 4.85 \\
\hline Cáncer de pulmón & 4 & 3.88 \\
\hline Cáncer de útero & 3 & 2.91 \\
\hline Carcinoma metastasico & 3 & 2.91 \\
\hline Hipertensión arterial & 3 & 2.91 \\
\hline Desnutrición proteica del adulto & 3 & 2.91 \\
\hline Shoque séptico & 3 & 2.91 \\
\hline Linforma de Hodgkin & 2 & 1.94 \\
\hline Cáncer de páncreas. & 2 & 1.94 \\
\hline Malformación congénita & 2 & 1.94 \\
\hline Alcoholismo crónico & 2 & 1.94 \\
\hline Cáncer de parótida derecha & 1 & 0.97 \\
\hline Cáncer de vejiga. & 1 & 0.97 \\
\hline Leucemia linfoblástica crónica. & 1 & 0.97 \\
\hline Insuficiencia cardiaca congestiva & 1 & 0.97 \\
\hline Cáncer de vesícula. & 1 & 0.97 \\
\hline Tumor de yeyuno. & 1 & 0.97 \\
\hline Tumor retroperitoneal. & 1 & 0.97 \\
\hline Enfermedad de Alzheimer & 1 & 0.97 \\
\hline
\end{tabular}

Fuente: Estadística de Hospital San Felipe.

mejorar las condiciones de vida de la población y obtener impactos de mayor eficacia como ser la esperanza de vida.

El análisis de la procedencia permite establecer que la accesibilidad geográfica es determinante para recibir la atención en el hospital, de tal forma que la demanda de los pacientes de Francisco Morazán representa más de la mitad de los datos analizados. En el informe presentado por la PNUD en el año 2010, se menciona que en Honduras todavía existe una gran brecha en el acceso a todo tipo de servicios públicos entre el área rural y el área urbana (PNUD, 2010).

Los datos por sexo, señalan que las mujeres son las que tienen una mayor necesidad de servicios de salud que los hombres, derivada pero no exclusivamente de su 
rol biológico en su reproducción y en mayor longevidad. Las mujeres se encuentran sobre representadas entre los pobres y por ello tienen menor acceso a una remuneración y a otros recursos para cuidar su salud, incluyendo los servicios de salud (Paz., 2004).

A nivel de edad, los rangos se definen a partir del medio día de vida hasta los 91 años. La media de edad para todo el grupo es de 61.9 años, menor al dato nacional de esperanza de vida que tiene el país para el periodo del 2010, que es de 73.1 año para toda la población y de 70.7 para hombres y de 75.6 para mujeres (BCIE, 2010).

Los datos de muerte perinatal son relevantes ya que se notificaron 4 en todo el año, con lo que se establece un aporte importante en un país que ha tenido antecedentes de mortalidad infantil superior al resto de los países de la Región Centroamericana. La reducción de las causas de mortalidad infantil se ha debido al aumento en la atención de los partos institucionales, al mejoramiento de las técnicas aplicadas y al aumento de las atenciones de las mujeres embarazadas en el lapso previo al parto. (PNUD, 2010), sin embargo, es importante establecer que las muertes que se presentaron en el periodo perinatal deben ser objetos de un análisis más específico considerando que la mortalidad perinatal es un indicador de los riesgos de muerte que permite tener un reflejo de la atención prenatal, durante el parto y el post parto actuando como demarcador tanto en la calidad del servicio de salud materno infantil y permite medir el estado de salud del producto durante los últimos dos meses de vida intrauterina y los primeros 6 días de vida extrauterina (Ticona \& Huanco, 2004).

Los datos de causas de muerte señalan al cáncer como la principal causa de muerte hospitalaria, de tal forma que al sumarizar todas las causas, representa el 63.5\%, es decir, más de la mitad de la causas analizadas. La OMS ha estimado que el cáncer no es un problema de países ricos, más del 70\% de las muertes por cáncer suceden en países de ingresos bajos y medianos, Honduras no es la excepción. Establece que más del 40\% de los cánceres son evitables y recomienda acciones de tipo preventivo y de autocuidado (OMS, 2006.).
También se encuentran descritas enfermedades crónicas que afectan al sistema cardíaco, v.g. la hipertensión arterial que está considerada como un marcador de riesgo más importante de morbilidad y mortalidad cardiovascular. Aunque la prevalencia se puede determinar de una forma relativamente fácil, no sucede lo mismo con la mortalidad relacionada con ella por lo que se requiere que se definan estrategias de prevención para lograr evitarla, aún cuando hay aspectos que no son controlables y que se encuentran definidos en las diferentes clasificaciones internacionales. (Espinoza Brito, 2012).

Las causales relacionadas con las enfermedades infecciosas, representa un indicador de la calidad de la atención médica que mide la eficiencia de un hospital (González \& Roig, 2011).

\section{Bibliografía}

- BCIE. (2010). Ficha estadística para Honduras.

- Espinoza Brito, A. D. (2012). Mortalidad por Hipertensión arterial. Tras la huella del "asesino silente". Finlay revista de enfermedades no transmisibles. Vol 2. No1. (2012).

- González, M. E., \& Roig, J. J. (2011). Relación entre mortalidad hospitalaria y sepsis durante 15 años. La Habana.

- OMS. (2006.). Cara a cara con la enfermedad cronica: el cáncer.

- Paz., O. G. (2004). Servicios de Salud con enfoque de Género en el Marco de la Atención Primaria de la Salud. OPS/OMS Washington.

- PNUD. (2010). Objetivos de Desarrollo del Milenio. Honduras 2010. Tercer Informe de País. Tegucigalpa.

- Salud, D. G. (2002). Estadística de Egresos Hospitalarios del Sector Público del Sistema Nacional de Salud,2000. México: Salud Pública de México./vol. 44.No.2. marzo-abril de 2002.

- Ticona, M., \& Huanco, D. (2004). Mortaliidad Perinatal. Lima: Rev Per Ginecol Obstet 2004; 50:61-71. 